\title{
Cold Allodynia after C2 Root Resection in Sprague-Daw- ley Rats
}

\author{
Daeyeong Chung, M.D., ${ }^{1}$ Dae-Chul Cho, M.D., Ph.D., ${ }^{2}$ Seong-Hyun Park, M.D., Ph.D., ${ }^{2}$ Kyoung-Tae Kim, M.D., Ph.D., ${ }^{2}$ \\ Joo-Kyung Sung, M.D., Ph.D., ${ }^{2}$ Younghoon Jeon, M.D., Ph.D. ${ }^{3}$ \\ Department of Neurosurgery, Cham Teun Teun Hospital, Daegu, Korea \\ Department of Neurosurgery, Kyungpook National University Hospital, Daegu, Korea \\ Department of Anesthesiology and Pain Medicine, ${ }^{3}$ School of Dentistry, Kyungpook National University, Daegu, Korea
}

Objective : The purpose of this study was to evaluate pain-related behaviors after bilateral C2 root resection and change in pain patterns in the suboccipital region in rats.

Methods : Male Sprague-Dawley rats were randomly assigned to three groups ( $\mathrm{n}=25 / \mathrm{group}$ ); näive, sham, and C2 resection. Three, 7, 10, and 14 days after surgery, cold allodynia was assessed using $20 \mu \mathrm{L}$ of $99.7 \%$ acetone. c-Fos and c-Jun were immunohistochemically stained to evaluate activation of dorsal horn gray matter in C2 segments of the spinal cord 2 hours, 1 day, 7 days, and 14 days after surgery.

Results : Three days after surgery, the response to acetone in the sham group was significantly greater than in the näive group, and this significant difference between the näive and sham groups was maintained throughout the experimental period $(p<0.05$ at $3,7,10$, and 14 days). Seven, 10 , and 14 days after surgery, the $C 2$ root resection group exhibited a significantly greater response to acetone than the näive group $(p<0.05)$, and both the sham and C2 resection groups exhibited significantly greater responses to acetone compared with 3 days after surgery. No significant difference in cold allodynia was observed between the sham and C2 root resection groups throughout the experimental period. Two hours after surgery, both the sham and C2 root resection groups exhibited significant increases in c-Fos- and c-Jun-positive neurons compared with the naive group ( $p=0.0021$ and $p=0.0358$ for the sham group, and $p=0.0135$ and $p=0.014$ for the $C 2$ root resection group, respectively). One day after surgery, both the sham and $C 2$ root resection groups exhibited significant decreases in c-Fos -positive neurons compared with two hours after surgery $(p=0.0169$ and $p=0.0123$, respectively), and these significant decreases in c-Fos immunoreactivity were maintained in both the sham and C2 root resection groups 7 and 14 days after surgery. The sham and C2 root resection groups presented a tendency toward a decrease in c-Jun-positive neurons 1, 7, and 14 days after surgery, but the decrease did not reach statistical significance.

Conclusion : We found no significant difference in cold allodynia and the early expression of c-Fos and c-Jun between the sham and $\mathrm{C} 2$ resection groups. Our results may support the routine resection of the $\mathrm{C} 2$ nerve root for posterior C1-2 fusion, but, further studies are needed.

Key Words : Cold allodynia · C2 root resection · Neuropathic pain · Posterior atlantoaxial fusion.

- Received : April 27, 2017 •Revised : September 3, 2017 •Accepted : September 6, 2017

-Address for reprints : Dae-Chul Cho, M.D., Ph.D.

Department of Neurosurgery, Kyungpook National University Hospital, 130 Dongdeok-ro, Jung-gu, Daegu 41944, Korea

Tel : +82-53-420-5649 Fax : +82-53-423-0504, E-mail : dccho@knu.ac.kr

This is an Open Access article distributed under the terms of the Creative Commons Attribution Non-Commercial License (http://creativecommons.org/licenses/by-nc/4.0) which permits unrestricted non-commercial use, distribution, and reproduction in any medium, provided the original work is properly cited. 


\section{INTRODUCTION}

Posterior atlantoaxial segmental instrumented fusion can be technically challenging, but it is a highly successful surgical option for craniocervical junction pathology. Recently, polyaxial screw and rod fixation of the C1 lateral mas and C2 pedicle has gained popularity ${ }^{10,14,16,22)}$. Goel et al. ${ }^{11-14)}$ reported that in cases of C1-2 instability with basilar invagination, posterior atlantoaxial fusion is feasible through intraoperative cervical traction, followed by distraction of the C1-2 facet joint, and subsequently, firm lateral mass fixation ${ }^{11,12,14)}$. They advocated that denuding the C1-2 articular cartilage and stuffing the bone graft within the $\mathrm{C} 1-2$ joint space can provide strong stability for $\mathrm{C} 1-2$ fixation. Based on their experience in treating more than 800 patients with atlantoaxial instability, most of them involving bilateral sectioning of the C2 root ganglion, they demonstrated that postoperative numbness in C2 dermatomes was well tolerated and did not lead to marked discomfort ${ }^{11-14)}$. Although some studies have advocated C2 root sectioning for C1 lateral mass screw insertion ${ }^{13,15,21)}$, C2 root resection for the placement of $\mathrm{C} 1$ lateral mass screws remains controversial.

Yeom et al. ${ }^{32)}$ recommended against routine C2 nerve root transection when performing atlantoaxial segmental screw fixation. In their experience, more than a quarter of patients experienced an increase in neuralgic pain following $\mathrm{C} 2$ nerve root trasection. Therefore, remaining uncertain is whether $\mathrm{C} 2$ root resection is appropriate for posterior $\mathrm{Cl}-2$ fusion, likely because of a lack of studies that have specifically investigatd whether it affects patient outcome.

Randomized human studies of experimental applications of C2 root resection during posterior $\mathrm{C} 1-2$ fixation are very limited because of ethical considerations. Therefore, there is the need for a practical animal model of $\mathrm{C} 2$ root resection to gain insights into the pathomechanism and treatment of suboccipital neuralgia after C2 resection. To our knowledge, no study has evaluated suboccipital pain after $\mathrm{C} 2$ root resection in animals.

In the present study, we evaluated pain-related behavior after bilateral C2 root resection to investigate changes in pain patterns in suboccipital regions in rats. We analyzed the immunoreactivity of c-Fos and c-Jun, markers of neuronal activation and response to noxious stimulation, within 2 weeks after $\mathrm{C} 2$ root resection ${ }^{2,3,17,25,31)}$.

\section{MATERIALS AND MATHODS}

All of the animal experiments were performed in accordance with the animal care guidelines of the National Institutes of Health, and were approved by the Institutional Animal Care Committee at Kyungpook National University Hospital (KNU 2016-99).

Seventy-five adult male Sprague-Dawley rats (11 weeks old) were purchased from Koatech Bio Inc. (Pyeongtaek, Korea) and acclimated to the laboratory environment for 1 week before the experiment. The rats were housed in an air-conditioned room (with 50\% relative humidity) under a 12-hour/12 hours light/dark cycle at $23 \pm 2{ }^{\circ} \mathrm{C}$ and given free access to food and tap water. The following week, the rats (at 12 weeks of age and weighing $340370 \mathrm{~g}$ ) were randomly and blindly assigned to three groups ( $n=25$ per group); naive, sham, and C2 resection. The rats were intraperitoneally anesthetized with a mixture of xylazine $(10 \mathrm{mg} / \mathrm{kg}$ ) and ketamine $(90 \mathrm{mg} / \mathrm{kg})$. The rats in the naive group were awakened after anesthesia and did not undergo surgery. The rats in the $\mathrm{C} 2$ root resection group underwent bilateral C2 root resection. Briefly, after the surgical site on the suboccipital and posterior neck region were shaved and prepared, the rats were placed prone on the operating table. A posterior midline skin incision was made over the upper cervical spine, and the posterior $\mathrm{C} 1$ arch and $\mathrm{C} 2$ lamina were exposed by dissecting the neck muscles. Careful dissection around the $\mathrm{C} 1-2$ joint was performed to find the $\mathrm{C} 2$ root ganglion just below the $\mathrm{Cl}$ posterior arch without any massive bleeding. Once exposed, the bilateral C2 roots were completely transected using microscissors at an area just immediately proximal to the dorsal root ganglion (Fig. 1). After surgery, the muscle, fascia, and skin were sutured using $4-0$ prolene sutures, and postoperative antibiotics were given subcutaneously (0.5 $\mathrm{mg} / \mathrm{kg}$ gentamicin). The rats in the sham group underwent an identical surgical procedure as the $\mathrm{C} 2$ root resection group but were not subjected to $\mathrm{C} 2$ root resection.

To avoid bias, the researcher, who conducted the behavioral test of cold allodynia, was blinded to the nature of the experimental manipulatios. Behavioral testing of cold allodynia was performed between 8:00 AM and 11:00 AM. On the experimental days, each rat was individually placed in a transparent plastic cage ( $25 \mathrm{~cm}$ width $\times 18 \mathrm{~cm}$ height $\times 35 \mathrm{~cm}$ length) in a quiet testing room for 30 minutes before behavioral measurements to allow the animal to adapt to the test environment. 

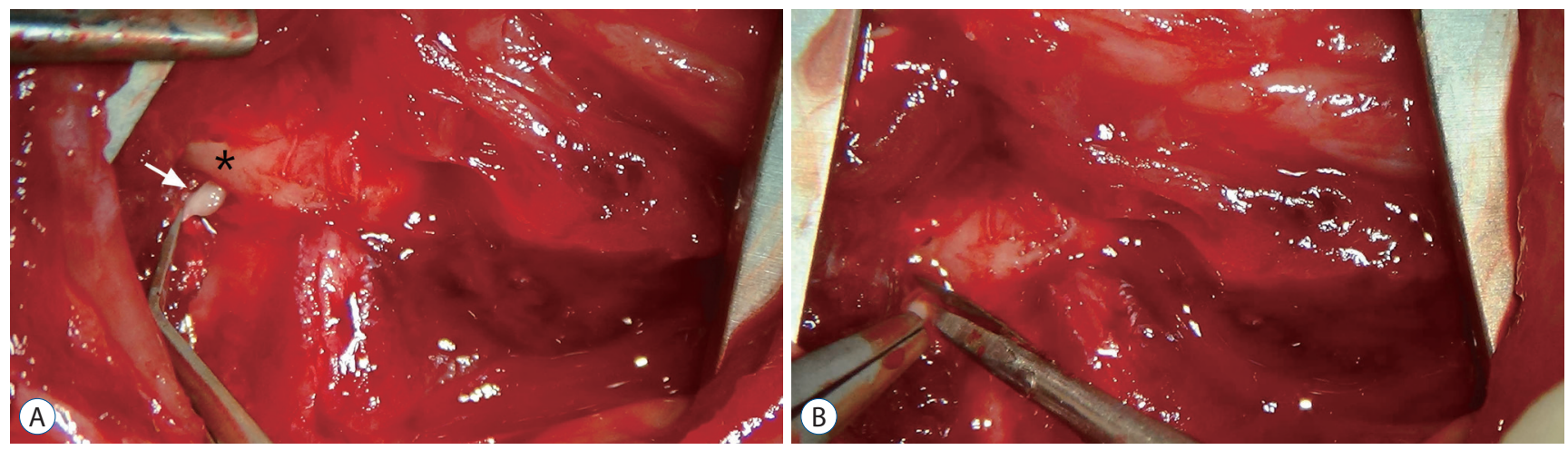

Fig. 1. After careful dissection of the posterior neck muscle, the $C 2$ root ganglion was revealed just below the $C 1$ posterior arch. $A$ : The white arrow indicates the $\mathrm{C} 2$ root ganglion. The asterisk indicates the posterior $\mathrm{C} 1 \mathrm{arch}$. B : Once exposed, the bilateral C2 roots were completely transected using microscissor in an area just proximal to the dorsal root ganglion.

For the behavioral test of cold allodynia, $20 \mu \mathrm{L}$ of $99.7 \%$ acetone was dropped onto the glabrous surface below both ears (Fig. 2), and the number of episodes of forepaw scratching and aggressive behavior, including severe head shaking was recorded for 1 minute. We repeated the behavioral test three times at 5 minutes intervals, and the number of responses was averaged. Cold allodynia was examined at 3, 7, 10, and 14 days after surgery.

For immunohistochemical staining, C2 segments of the spinal cord were extractred from the naive, sham, and C2 resection groups ( $\mathrm{n}=46 /$ group) 2 hours, 1 day, 7 days, and 14 days after surgery. The rats were then transcardially perfused with $150 \mathrm{~mL}$ of normal saline, followed by $4 \%$ paraformaldehyde in $0.1 \mathrm{M}$ phosphate-buffered saline (PBS; $\mathrm{pH}$ 7.4) under deep anesthesia with a lethal dose of urethane (Sigma-Aldrich, St. Louis, MO, USA). Samples were postfixed in 4\% paraformaldehyde overnight and then gradually dehydrated in ethanol and embedded in paraffin. Representative sections of the dorsal root entry zone were sliced into $3 \mu \mathrm{m}$-thick sections in the horizontal plane and mounted onto $0.02 \%$ poly-L-lysinecoated slides. Deparaffinized sections were quenched in hydrogen peroxide solution ( $10 \% \mathrm{H}_{2} \mathrm{O}_{2}$ in PBS for 10 minutes), retrieved in citric acid ( $\mathrm{pH}$ 6.0) for 20 minutes at microwave, incubated in $50 \mathrm{mM}$ ammonium chloride $\left(\mathrm{NH}_{4} \mathrm{Cl}\right)$ for $30 \mathrm{~min}$ utes at room temperature, and blocked with blocking solution ( $1 \%$ bovine serum albumin, $0.2 \%$ gelatin, $0.05 \%$ saponin in PBS, and 5\% normal goat serum) for 1 hour at room temperature. The sections were then incubated overnight at $4^{\circ} \mathrm{C}$ with cFos or c-Jun primary antibody (1:100; Santa Cruz Biotechnology, Santa Cruz, CA, USA) in PBS. The next day, after rinsing with PBS three times, the sections were incubated in biotinyl-

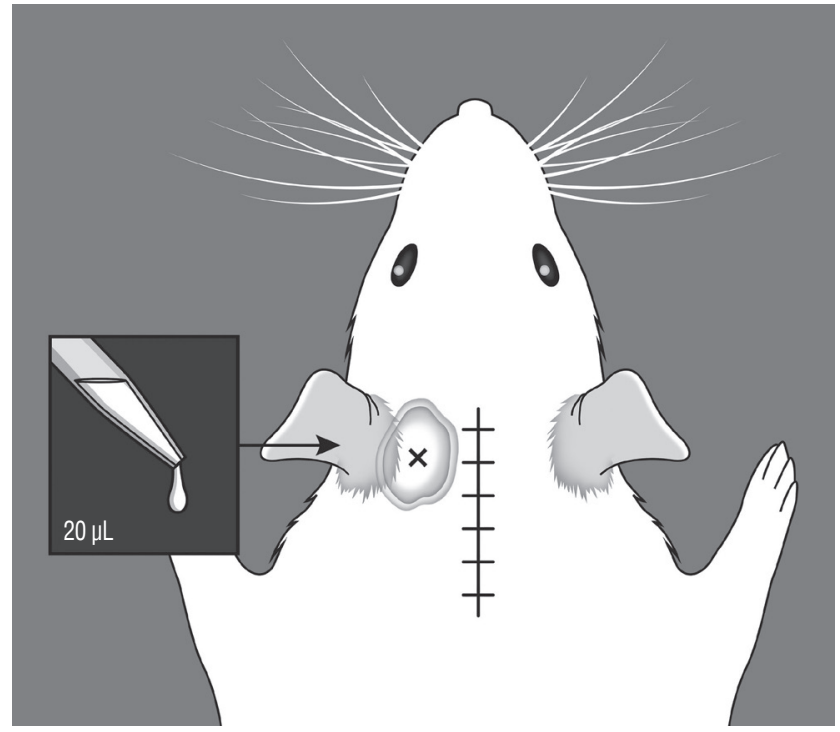

Fig. 2. Illustration of cold allodynia testing. A volume of $20 \mu \mathrm{L}$ of $99.7 \%$ acetone was dropped onto the glabrous surface below the ears. The acetone percolated through the suboccipital area, not cross the midline suture site.

ated goat anti-rabbit secondary antibody $(1: 200$ in PBS) and subsequently in an avidin-horseradish peroxide solution. Immunolabeling was visualized with 0.05 3, 3'-diaminobenzidine hydrochloride plus $0.3 \%$ hydrogen peroxide in PBS. The sections were then dehydrated with ethanol and xylene before mounting under coverslips with Permount.

Hematoxylin-eosin staining was used to determine morphological changes in the naive, sham, and C2 resection groups. Deparaffinized sections were dipped in Harris hematoxylin (Sigma-Aldrich) for 5 minutes, neutralized with $0.5 \% \mathrm{HCl}$ and $0.05 \%$ ammonia water, dipped in eosin for 45 seconds, dehydrated in ethanol and mounted on the glasse slides. Photos of the stained sections were taken using a Zeiss Axioplan micro- 


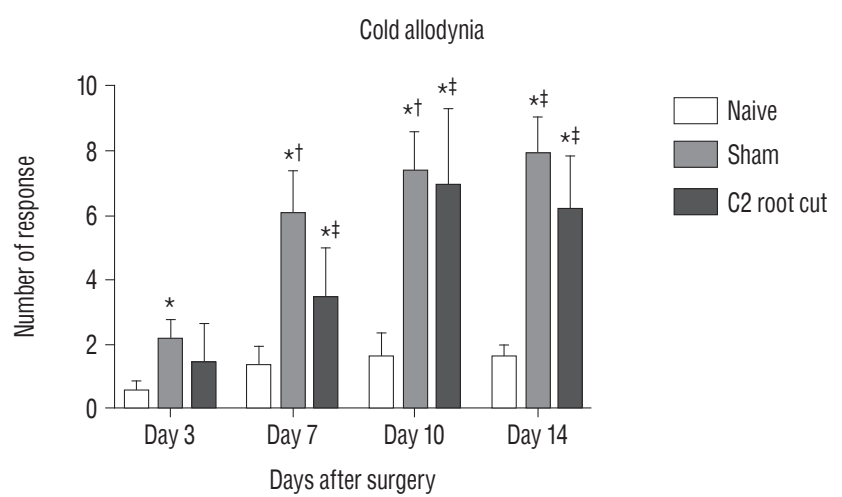

Fig. 3. Evaluation pain-related behavior (cold allodynia) for 2 weeks after surgery. The data are expressed means \pm standard error of mean. ${ }^{*} p<0.05$, compared with naive group. ${ }^{\dagger} p<0.05$, compared with sham group 3 days after surgery. ${ }^{\ddagger} p<0.05$, compared with $C 2$ root resection group 3 days after surgery. No significant difference in cold allodynia was found between the sham and $\mathrm{C} 2$ root resection groups at each time point throughout the experimental period.

scope (Carl Zeiss Meditec Inc., Jena, Germany). The images were viewed on a computer monitor using a Zeiss Plan-Apochromat 100X objective and Zeiss AxioCam HRc digital camera (Carl Zeiss Meditec Inc.). The analysis of c-Fos and c-Jun immunoreactivity was quantified by determining the number of $\mathrm{c}$ Fos- and c-Jun- positive nuclei in dorsal horn gray matter of the spinal cord. The regions of gray matter corresponded to Rexed superficial laminae I-III and deeper laminae IV-VI (Fig. 3).

\section{Statistical analysis}

All of the statistical analyses were performed using Prism 7 software (GraphPad, La Jolla, CA, USA). The data are expressed as mean \pm standard error of mean. Repeated measure analysis of variance was for comparison between groups. Values of $p<0.05$ were considered statistically significant.

\section{RESULTS}

Postoperative pain-related behavior (i.e., cold allodynia) in response to $99.7 \%$ acetone showed difference among groups 3 , 7, 10, and 14 days after surgery (Fig. 3). The naive group did not exhibit any significant differences in the response to acetone throughout the experiment period. The patterns of cold allodynia in both the sham and C2 resection groups were similar during the experiment. Three days after surgery, the response to acetone in the sham group was significantly great- er than in the naive group, and this significant difference between the naive and sham groups was maintained throughout the experimental period ( $p<0.05$ at $3,7,10$, and 14 days). The $\mathrm{C} 2$ root resection group exhibited a greater response to acetone 3 days after surgery, but this response did not reach statistical significance compared with the naive group $(p>0.05)$. Seven, 10, and 14 days after surgery, the C2 root resection group exhibited a significantly greater response to acetone compared with the naive group $(p<0.05)$. Seven, 10 , and 14 days after surgery, both the sham and $\mathrm{C} 2$ resection groups exhibited significantly greater responses to acetone compared 3 days after surgery. However, no significant difference in cold allodynia was observed between the sham and C2 root resection groups at each time point throughout the experimental period.

Histologic examination of C2 segments of spinal cords did not show any morphological changes in all three groups throughout the experimental periods (Fig. 4). Immunohistochemical staining of c-Fos and c-Jun in C2 segments of the spinal cord showed that, in the naive group, there were no significant changes in c-Fos and c-Jun immunoreactivity throughout the experimental period ( $p>0.05$, Figs. 5 and 6). Two hours after surgery, both the sham and $\mathrm{C} 2$ root resection groups exhibited significant increases in c-Fos- and c-Jun-positive neurons compared with the naive group $(p=0.0021$ and $p=0.0358$ in the sham group, and $p=0.0135$ and $p=0.014$ in the $\mathrm{C} 2$ root resection group, respectively). One day after surgery, both the sham and C2 root resection groups exhibited significant decreases in c-Fos-positive neurons compared with 2 hours after surgery ( $p=0.0169$ and $p=0.0123$, respectively), and these significant decreases were maintained in both the sham and C2 root resection groups 7 and 14 days after surgery.

Both the sham and C2 root resection groups presented a tendency toward a decrease in c-Jun-positive neurons 1,7 , and 14 days after surgery, but this decrease did not reach statistical significance.

\section{DISCUSSION}

Since the first introduction by Harms and Melcher polyaxial screw and rod fixation of the $\mathrm{C} 1$ lateral mass and $\mathrm{C} 2$ pedicle has gained popularity for craniovertebral junction pathology $^{10,14,16,22)}$. Originally, Harms and Melcher ${ }^{16)}$ did not sacrifice 

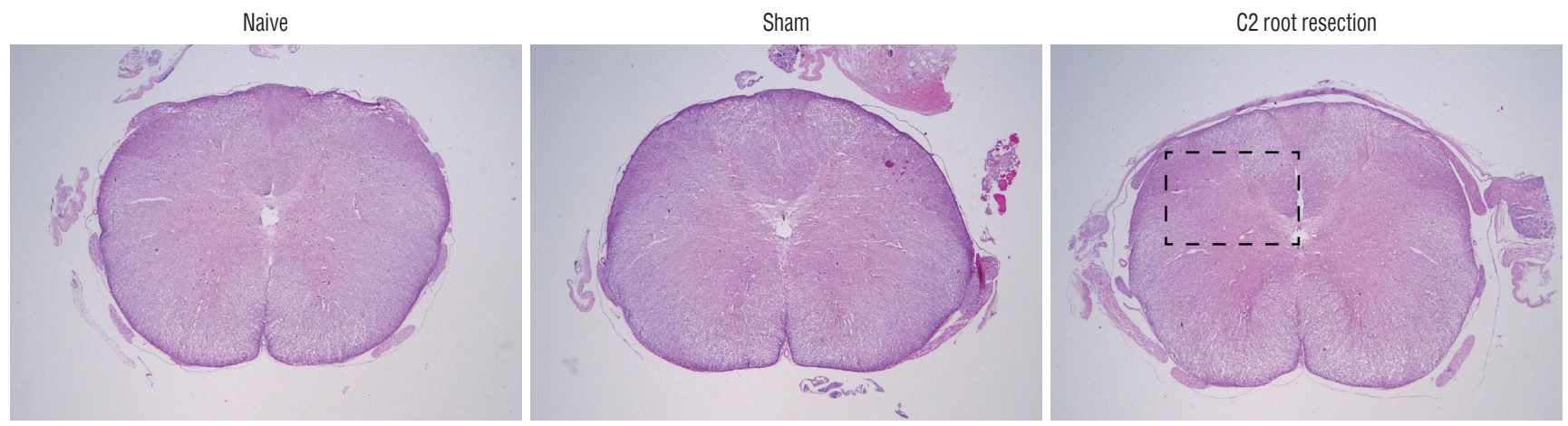

Fig. 4. Representative spinal cord sections stained with hematoxylin and eosin at C2 segment of spinal cord at 1 day after experiment. Black box shows the area for quantification of c-Fos- and c-Jun-positive nuclei in dorsal horn gray matter of Fig. 5 . Scale bar=500 $\mu$ m; $20 \times$ magnification.

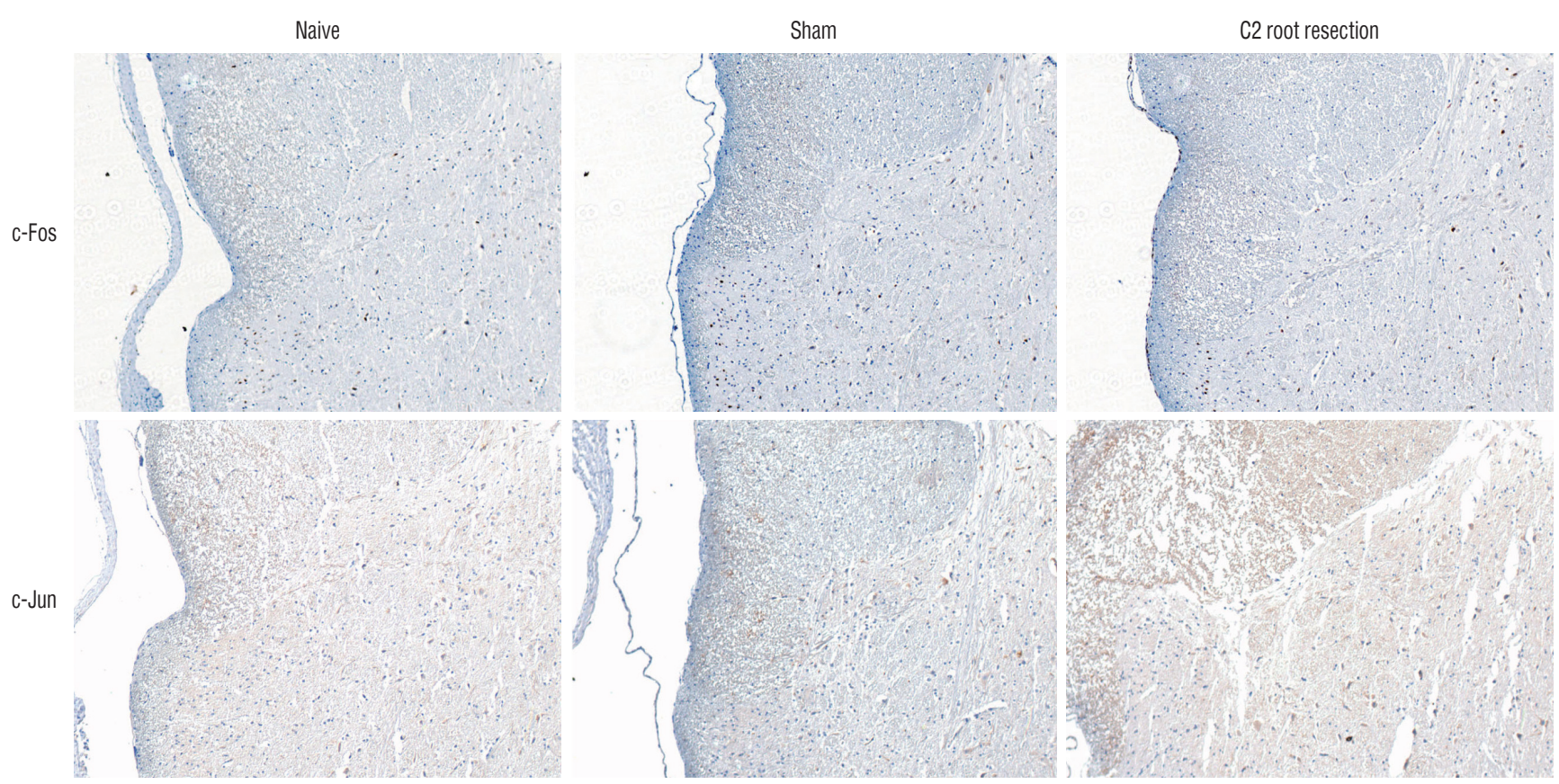

Fig. 5. Immunohistochemical staining of c-Fos and c-Jun. Representative photographs of c-Fos- and c-Jun-postive immunoreactivity cells from dorsal horn gray matter of $\mathrm{C} 2$ segments of spinal cord at 1 day after experiment. 100xmagnification.
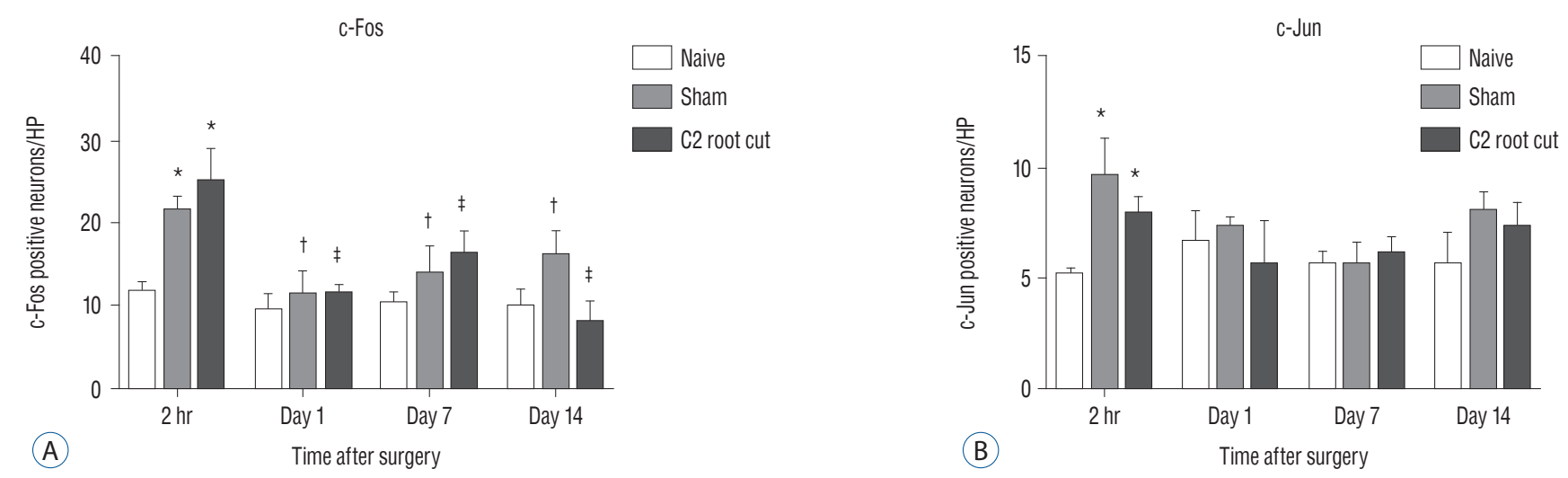

Fig. 6. Immunohistochemical staining of C-Fos (A) and c-Jun (B) in C2 segments of the spinal cord. ${ }^{*} p<0.05$, compared with naive group. ${ }^{\dagger} p<0.05$, compared with sham group 2 hours after surgery. ${ }^{\ddagger} p<0.05$, compared with $C 2$ root resection group 2 hours after surgery. 
the C2 nerve root and instead used a C2 screw with a smooth unthreaded portion to minimize irritation of the $\mathrm{C} 2$ nerve root. They achieved fusion in $100 \%$ of their series of 37 patients and did not encounter any neural complications including C2 neuralgia. However, recent studies have advocated routine transection of the $\mathrm{C} 2$ root for posterior $\mathrm{C} 1-2$ fusion, because it can improve exposure of the screw insertion sites and $\mathrm{C} 1-\mathrm{C} 2$ facet joints, increase the fusion bed area, and allow the easier control of bleeding from the venous plexus at $\mathrm{C} 1-\mathrm{C} 2^{9,11,12,15)}$. Goel et al. ${ }^{11-14)}$ demonstrated that postoperative numbness in $\mathrm{C} 2$ dermatomes after bilateral section of the $\mathrm{C} 2$ ganglion is well tolerated and does not lead to any marked discomfort. The numbness and heaviness on the back of the head progressively lessens in intensity and extent with time ${ }^{11-14)}$. In a recent study by Hamilton et al. ${ }^{15)}, 80 \%$ of patients in their series had preoperative occipital neuralgia. In all of these patients, this neuralgia was relieved by $\mathrm{C} 1-2$ instrumented arthrodesis with $\mathrm{C} 2$ neurectomy.

However, remaining controversial is whether preservation or transection of the $\mathrm{C} 2$ nerve root results in a reduction of postoperative occipital neuralgia. A recent study by Yeom et al. ${ }^{32)}$ showed that more than a quarter of the patients in their series experienced an increase in neuralgic pain following C2 nerve root transection. Therefore, these authors argued against routine $\mathrm{C} 2$ nerve root transection when performing atlantoaxial segmental screw fixation ${ }^{32)}$. These opposing viewpoints regarding suboccipital neuralgia after $\mathrm{C} 2$ nerve root transection raised the need to perform further well-designed prospective randomized controlled studies.

Animal models provide a systematic and well-controlled experimental environment, and are useful for identifying potential therapeutic targets for neuropathic pain ${ }^{28)}$. In the present study, we used Sprague-Dawley rats to investigate behavioral responses to pain in the suboccipital area after bilateral $\mathrm{C} 2$ root resection.

Allodynia is defined as a painful response to normally innocuous stimuli. Hyperalgesia is defined as increased in the pain response to normally painful stimuli. Both allodynia and hyperalgesia are major components of neuropathic pain, which refers to pain that originates from pathology of the nervous system, including the central nervous system (e.g., spinal cord) and peripheral nerves. In neuropathic pain, tissue damage directly affects the nervous system, resulting in the generation of ectopic discharges that bypass transduction ${ }^{4,6,24)}$. Per- sistent postoperative pain can be a consequence of nerve injury during surgery, and abnormal signals arise from both injured axons and intact nociceptors that share the innervation territory of the injured nerve ${ }^{4,28)}$.

In the present study, we evaluated cold allodynia in the suboccipital area in Sprague-Dawley rats after bilateral C2 root resection. Both the sham and $\mathrm{C} 2$ resection groups presented similar patterns of cold allodynia during the experiment. Three days after surgery, the response to acetone in the sham group was greater than in the naive group, and this difference was maintained throughout the experimental period. The C2 root resection group did not exhibit a significant difference in cold allodynia compared with the sham group at any of the time points after surgery. Although cold allodynia in an animal model dose not soley reflect the overall characteristics of neuropathic pain, our results support routine transection of the $\mathrm{C} 2$ nerve root for posterior $\mathrm{C} 1-2$ fusion, as suggested by Goel et al. ${ }^{11-14)}$. Among the various animal models of neuropathic pain ${ }^{7,18,23,24)}$, the spared nerve injury model was first developed by Decosterd and Woolf in 2000 in Sprague-Dawley rats to enhance the reproducibility of injury and behavioral responses to partial nerve injury ${ }^{7)}$. In rats, cold allodynia developed immediately after spared nerve injury, and was maintained for several months during the observation period, as well as other components of neuropathic pain such as heat hyperalgesia, mechanical allodynia and mechanical hyperalgesia $^{7)}$. With regard to cold allodynia, the present results were consistent with this previous study.

The present study used two markers of neuronal activation; $c$-Fos (the protein product of the immediate-early gene, $c$-fos), and c-Jun (the protein product of the immediate-early gene, $c$ jun). These two markers have been widely used to study the neural correlates of nociception, neuronal activation and noxious stimulation ${ }^{2,3,17,25,31)}$. A recent study by Li et al. ${ }^{25)}$ reported that the number of c-Fos- and c-Jun-positive neurons in ipsilateral C5-7 segments significantly increased 2 and 4 hours after $\mathrm{C} 7$ nerve root rhizotomy compared with the sham group. The localization of c-Fos- and c-Jun-positive neurons in C5-7 gray matter was mainly found in lamina IX of the anterior horn and laminae I-II of the dorsal horn of the spinal cord. The number of c-Fos- and c-Jun-positive neurons in C5-7 gray matter significantly decreased 4 hours after surgery compared with 2 hours after surgery. Our results also showed a similar pattern of c-Fos and c-Jun expression after C2 root resection. 
In the present study, the $\mathrm{C} 2$ root resection group exhibited a significant increase in c-Fos and c-Jun expression 2 hours after surgery compared with the naive group, and a significant decrease in c-Fos expression 1 day after surgery compared with 2 hours after surgery. These findings are consistent with the previous study. In the present study, the sham group also exhibited a significant increase in c-Fos- and c-Jun-positive neurons, which is opposite to the findings of Li et al. ${ }^{25)}$. These diametrically opposing results in the sham group reflect the different animal species or different models used.

In humans, the anatomy of the C2 nerve root and its ganglion has been studied in detail. The $\mathrm{C} 2$ ganglion lies in the intervertebral space between $\mathrm{C} 1$ and $\mathrm{C} 2$, bordered by the posterior arch of the atlas, lamina of the axis, and atlantoaxial joint. The dorsal ramus of the $\mathrm{C} 2$ spinal nerve emerges between the posterior arch of the atlas and lamina of the axis, and the medial branches of the ramus terminate as the greater occipital nerve. After piercing the trapezius aponeurosis, the greater occipital nerve travels with the scalp as far anterior as the vertex of the skull ${ }^{8,26,29,30)}$. However, no data are available regarding the anatomy of the $\mathrm{C} 2$ nerve root and its innervated dermatomes in the suboccipital area in animals. We assumed that rats also have similar passages and innervations of the $\mathrm{C} 2$ nerve root. Therefore, we applied acetone to the glabrous surface below both ears. Acetone $(20 \mu \mathrm{L})$ percolated through the suboccipital area, but did not cross the midline suture site. We supposed that if the acetone crossed the midline suture site, then this would cause additional nociceptive incisional pain instead of neuropathic pain. Our preliminary study confirmed that $20 \mu \mathrm{L}$ acetone was sufficient to percolate through the suboccipital area in rats, and not cross the midline suture site (data not shown).

To test pain-related behavior in animals, most studies manipulate the plantar surface of the hindpaw, where there is no fur $^{1,3,5,19,20,27,28)}$. In the present study, although the surgical site in the suboccipital and posterior neck region was thoroughly shaved after anesthesia, minute amount of fur grew several days after shaving, which may hinder the precise testing of cold allodynia. We did not shave the region again, because, we thought that re-shaving with repeated anesthesia would be stressful to the animals, and thus affect pain-related behavior.

In the present study, we did not evaluate mechanical allodynia using a series of von Frey filaments, which is most commonly used to test pain-related behavior in animal models of neuropathic pain ${ }^{1,20)}$. To our knowledge, no reference data are available regarding mechanical allodynia after $\mathrm{C} 2$ root resection in animals. Mechanical allodynia testing in rat would be difficult to condut in the suboccipital region rather than the hindpaw.

\section{CONCLUSION}

The present study found no significant difference in cold allodynia and early expression of c-Fos and c-Jun between the sham and C2 resection groups. Our results may support the routine resection of the $\mathrm{C} 2$ nerve root for posterior $\mathrm{C} 1-2 \mathrm{fu}-$ sion, but further studies are needed.

\section{CONFLICTS OF INTEREST}

The authors have no financial conflicts of interest.

\section{INFORMED CONSENT}

This type of study does not require informed consent.

\section{References}

1. Ahn DK, Lim EJ, Kim BC, Yang GY, Lee MK, Ju JS, et al. : Compression of the trigeminal ganglion produces prolonged nociceptive behavior in rats. Eur J Pain 13 : 568-575, 2009

2. Berrocal $Y A$, Pearse DD, Andrade CM, Hechtman JF, Puentes R, Eaton $\mathrm{MJ}$ : Increased spinal c-Fos expression with noxious and non-noxious peripheral stimulation after severe spinal contusion. Neurosci Lett 413 : 58-62, 2007

3. Broude $E$, McAtee M, Kelley MS, Bregman BS : c-Jun expression in adult rat dorsal root ganglion neurons, differential response after central or peripheral axotomy. Exp Neurol 148 : 367-377, 1997

4. Campbell JN, Meyer RA : Mechanism of neuropathic pain. Neuron $\mathbf{5 2}$ : 77-92, 2006

5. Catheline G, Le Guen S, Honoré P, Besson JM : Are there long-term changes in the basal or evoked Fos expression in the dorsal horn of the spinal cord of the mononeuropathic rat? Pain 80 : 347-357, 1999

6. Cohen SP, Mao J : Neuropathic pain: mechanisms and their clinical implications. BMJ 348 : $f 7656,2014$

7. Decosterd I, Woolf CJ : Spared nerve injury: an animal model of persistent peripheral neuropathic pain. Pain 87 : 149-158, 2000 
8. Ducati A : Nerves are not made to be cut. World Neurosurg 78 : $601-$ 602, 2012

9. Elliott RE, Kang MM, Smith ML, Frempong-Boadu A : C2 nerve root sectioning in posterior atlantoaxial instrumented fusions: a structured review of literature. World Neurosurg $78:$ 697-708, 2012

10. Elliott RE, Tanweer O, Boah A, Morsi A, Ma T, Smith ML, et al. : Atlantoaxial fusion with screw-rod constructs: meta-analysis and review of literature. World Neurosurg 81 : 411-421, 2014

11. Goel $A$ : Treatment of basilar invagination by atlantoaxial joint distraction and direct lateral mass fixation. J Neurosurg Spine 1 : 281-286, 2004

12. Goel A : Cervical ganglion 2 (CG2) neurectomy: a window to the atlantoaxial joint. World Neurosurg 78 : 78-79, 2012

13. Goel A, Desai KI, Muzumdar DP : Atlantoaxial fixation using plate and screw method: a report of 160 treated patients Neurosurgery 51 : 1351-1356; discussion 1356-1357, 2002

14. Goel A, Laheri $V$ : Plate and screw fixation for atlantoaxial subluxation. Acta Neurochir (Wien) $129:$ 47-53, 1994

15. Hamilton DK, Smith JS, Sansur CA, Dumont AS, Shaffrey Cl : C-2 neurectomy during atlantoaxial instrumented fusion in the elderly: patient satisfaction and surgical outcome. J Neurosurg Spine 15 : 3-8, 2011

16. Harms J, Melcher RP : Posterior C1-C2 fusion with polyaxial screw and rod fixation. Spine (Phila Pa 1976) 26 : 2467-2471, 2001

17. Harris JA : Using c-Fos as a neural marker of pain. Brain Res Bull 45 : 1-8, 1998

18. Jaggi AS, Jain V, Singh $N$ : Animal models of neuropathic pain. Fundam Clin Pharmacol 25 : 1-28, 2011

19. Jeon HJ, Han SR, Park MK, Yang KY, Bae YC, Ahn DK : A novel trigeminal neuropathic pain model: compression of the trigeminal nerve root produces prolonged nociception in rats. Prog Neuropsychopharmacol Biol Psychiatry 38 : 149-158, 2012

20. Jeon Y, Kim CE, Jung D, Kwak K, Park S, Lim D, et al. : Curcumin could prevent the development of chronic neuropathic pain in rats with peripheral nerve injury. Curr Ther Res Clin Exp 74 : 1-4, 2013

21. Kang MM, Anderer EG, Elliott RE, Kalhorn SP, Frempong-Boadu A : C2 nerve root sectioning in posterior C1-2 instrumented fusions. World Neurosurg $78: 170-177,2012$
22. Kim IS, Hong JT, Sung JH, Byun JH : Verical reduction using atlantoaxial facet spacer in basilar invagination with atlantoaxial instability. J Korean Neurosurg Soc 50 : 528-531, 2011

23. Kim KJ, Yoon YW, Chung JM : Comparison of three rodent neuropathic pain models. Exp Brain Res 113 : 200-206, 1997

24. Klusáková I, Dubový P : Experimental models of peripheral neuropathic pain based on traumatic nerve injuries - an anatomical perspective. Ann Anat 191 : 248-259, 2009

25. Li H, Li Q, Xie K, Feng S, Wang P, Ma X : Expression of c-Fos and c-Jun in adjacent cervical spinal cord segments following $\mathrm{C} 7$ nerve root rhizotomy in rats: indication of a neural pathway between adjacent cervical spinal cord segments. Exp Ther Med 6 : 373-377, 2013

26. Loukas M, El-Sedfy A, Tubbs RS, Louis RG Jr, Wartmann CT, Curry B, et al. : Identification of greater occipital nerve landmarks for the treatment of occipital neuralgia. Folia Morphol (Warsz) 65 : 337-342, 2006

27. McCoy ES, Zylka MJ : Enhanced behavioral responses to cold stimuli following CGRP $\alpha$ sensory neuron ablation are dependent on TRPM8. Mol Pain $10: 69,2014$

28. Sideris A, Norcini M, Blanck TJ, Reico-Pinto E : Temporal and qualitative differences in the development of allodynic behaviors between mice and rats in a peripheral nerve injury model. Pain Studies and Treatment $2: 121,2014$

29. Vanelderen $P$, Lataster $A$, Levy $R$, Mekhail $N$, van Kleef $M$, Van Zundert J : Occipital neuralgia. Pain Pract 10 : 137-144, 2010

30. Vital JM, Grenier F, Dautheribes M, Baspeyre H, Lavignolle B, Sénégas J : An anatomic and dynamic study of the greater occipital nerve ( $\mathrm{n}$. of Arnold). Applications to the treatment of Arnold's neuralgia. Surg Radiol Anat $11: 205-210,1989$

31. Wang TT, Yuan WL, Ke Q, Song XB, Zhou X, Kang Y, et al. : Effects of electro-acupuncture on the expression of c-jun and c-fos in spared dorsal root ganglion and associated spinal laminae following removal of adjacent dorsal root ganglia in cats. Neuroscience 140 : 1169-1176, 2006

32. Yeom JS, Buchowski JM, Kim HJ, Chang BS, Lee CK, Riew KD : Postoperative occipital neuralgia with and without $C 2$ nerve root transection during atlantoaxial screw fixation: a post-hoc comparative outcome study of prospectively collected data. Spine J $13:$ 786-795, 2013 